\title{
HTP-1 coordinates synaptonemal complex assembly with homolog alignment during meiosis in C. elegans
}

\author{
Florence Couteau and Monique Zetka ${ }^{1}$ \\ Department of Biology, McGill University, Montreal, QC H3A 1B1 Canada
}

\begin{abstract}
During meiosis, the mechanisms responsible for homolog alignment, synapsis, and recombination are precisely coordinated to culminate in the formation of crossovers capable of directing accurate chromosome segregation. An outstanding question is how the cell ensures that the structural hallmark of meiosis, the synaptonemal complex (SC), forms only between aligned pairs of homologous chromosomes. In the present study, we find that two closely related members of the him-3 gene family in Caenorhabditis elegans function as regulators of synapsis. HTP-1 functionally couples homolog alignment to its stabilization by synapsis by preventing the association of SC components with unaligned and immature chromosome axes; in the absence of the protein, nonhomologous contacts between chromosomes are inappropriately stabilized, resulting in extensive nonhomologous synapsis and a drastic decline in chiasma formation. In the absence of both HTP-1 and HTP-2, synapsis is abrogated per se and the early association of SC components with chromosomes observed in htp-1 mutants does not occur, suggesting a function for the proteins in licensing SC assembly. Furthermore, our results suggest that early steps of recombination occur in a narrow window of opportunity in early prophase that ends with SC assembly, resulting in a mechanistic coupling of the two processes to promote crossing over.
\end{abstract}

[Keywords: Meiosis; synapsis; homolog alignment; recombination; C. elegans]

Supplemental material is available at http://www.genesdev.org.

Received June 21, 2005; revised version accepted September 21, 2005.

Sexual reproduction requires the halving of the genome complement of germ cells, a feat that is accomplished by a single round of DNA replication followed by two successive divisions known as meiosis. Homologous chromosomes, each consisting of a pair of tightly associated chromatids, are segregated during the first round, while sister chromatids are segregated during the second. During the first division, the proper orientation of homologous chromosomes pairs on the spindle and their subsequent accurate segregation rely on the formation of physical linkages (chiasmata) resulting from crossover recombination between them. For decades, the formation of these crossovers has been linked to another structural landmark of meiosis, the synaptonemal complex $(\mathrm{SC})$ - a tripartite proteinaceous structure that is assembled between paired chromosomes.

The function of the SC in chromosome pairing has been clearly demonstrated in Caenorhabditis elegans, where the initial alignment of homologs and synapsis between them are genetically separable steps requiring

${ }^{1}$ Corresponding author.

E-MAIL monique.zetka@mcgill.ca; FAX (514) 398-5069.

Article and publication are at http://www.genesdev.org/cgi/doi/10.1101/ gad.1348205. distinct chromosome features. Chromosomes attain early alignment in the absence of SYP-1 and SYP-2, two putative SC components required for its formation, but this alignment is progressively lost as pachytene progresses, indicating that synapsis is essential for its stabilization (MacQueen et al. 2002; Colaiácovo et al. 2003). Synapsis is structurally coordinated with homolog alignment through HIM-3, a noncohesin component of meiotic chromosome axes that is required for both processes (Zetka et al. 1999; Couteau et al. 2004). In the absence of HIM-3, sister-chromatid cohesion and chromatin condensation are not detectably affected, but chromosomes fail to align and SC components like SYP-1 and SYP-2 form nuclear aggregates; however, recombination is initiated on time and at wild-type levels, indicating that early alignment and synapsis are not required for early steps in the recombination pathway (Couteau et al. 2004).

In addition to its role in stabilizing chromosome pairing, the SC is universally involved in the maturation of recombination intermediates to the chiasmata that will maintain the homologs in a physically paired configuration after desynapsis and direct their orderly disjunction at the first meiotic division (Walker and Hawley 2000). While the series of events leading to the formation of 
crossovers has been described at the DNA level (for review, see Villeneuve and Hillers 2001), dissecting their timing and mechanistic relationship to the dramatic structural changes that meiotic chromosomes undergo during prophase has remained a complex problem.

The SC is remarkably conserved at the ultrastructural level; two dense lateral elements (LE) corresponding to a pair of chromosome axes about which replicated sister chromatids are organized are separated by a less dense, striated central element of $\sim 100 \mathrm{~nm}$ composed of a series of transverse filaments (TF) (for review, see von Wettstein et al. 1984; Page and Hawley 2004). Lateral elements are derived from the axial elements of chromosomes that assemble between sister chromatids, and the known components of the LE are a diverse group of proteins with roles in homolog pairing, recombination, and TF assembly (Page and Hawley 2004). Putative TF constituents that have been identified from evolutionarily divergent species share little sequence homology, but have significant similarities at the level of secondary structure and organization (for review, see Hunter 2003). Most notably, TF proteins are characterized by globular termini separated by long, rod-like coiled-coils that interact through head-to-head associations to form homodimers that span the central region. The $\mathrm{C}$ terminus has been identified as critical for localization to chromosomes (Tung and Roeder 1998) and assembly of higherorder structures (Ollinger et al. 2005), suggesting that the association of the $\mathrm{C}$ termini of TF homodimers with the lateral elements is an early step of SC assembly, followed by the association of the $\mathrm{N}$ termini and rod domains of dimers on opposing lateral elements to form the central element. TF proteins show a remarkable propensity for self-association manifesting itself as polycomplex formation (Goldstein 1987) when expressed in mitotic cells (Ollinger et al. 2005) or overexpressed in meiotic cells (Sym and Roeder 1995), and when unable to associate with chromosomes in axis-defective mutants (Couteau et al. 2004). In some cases, polycomplexes can also be detected in prepachytene nuclei during normal meioses; in C. elegans, the putative TF proteins SYP-1 and SYP-2 are first detected as single aggregates in nuclei entering the meiotic cell cycle, followed by their localization to chromosomes as prophase progresses (MacQueen et al. 2002; Colaiácovo et al. 2003). At the ultrastructural level, polycomplexes bear striking similarity to SC, attesting to the potency and highly ordered nature of the self-assembly reaction (Dong and Roeder 2000; Ollinger et al. 2005). How the meiotic cell controls this polymerization to result in SC formation between chromosomes rather than in nuclear polycomplexes remains an outstanding question.

The efficient self-assembly of TF components into SClike structures poses an additional problem; how is synapsis between chromosomes controlled? SC formation is not dependent on DNA sequence homology and can readily form between nonhomologous chromosomes or chromosome segments (Goldstein 1986; Leu et al. 1998; Pawlowski et al. 2004). During meiosis, however, SC assembly is coordinated with the alignment of homolo- gous chromosomes such that synapsis is restricted to identical chromosome pairs that are aligned along their lengths (Zickler and Kleckner 1998). Given the ready polymerization of the central element, synapsis between homologous chromosomes is likely to be enforced at the level of initiation. In some organisms, SC assembly is dependent on double-strand break (DSB) formation, a conserved mechanism of meiotic recombination initiation thought to be catalyzed by the Spo11 topoisomerase (Keeney 2001). In the yeast Saccharomyces cerevisiae, for example, the coordination of homolog alignment and SC formation is promoted by events in the DSB repair pathway; early intermediates in the process are involved in homolog recognition and alignment (Peoples-Holst and Burgess 2005), and subsequently give rise to crossover products that are the sites of synapsis initiation (Fung et al. 2004). This mechanism is not universal, however, since in other organisms like Drosophila and C. elegans, synapsis does not require Spo-11 activity or recombination (Dernburg et al. 1998; McKim et al. 1998), and instead, SC formation is thought to initiate at distinct chromosomal sites (Zetka and Rose 1995; Sherizen et al. 2005). Regardless of the mechanism used to restrict the site of synapsis initiation, however, how SC components are prevented from associating with chromosome axes until homolog recognition and alignment have been attained is unknown.

In the present study, we investigate the function of $h t p-1$ and $h t p-2$ (him three paralog), two closely related genes that encode paralogs of the $C$. elegans meiosisspecific chromosome axis component HIM-3. Our analyses have allowed us to address how the natural proclivity of SC components for self-assembly, regardless of DNA homology, is controlled by identifying HTP-1 as a key component of the mechanism preventing the stabilization of nonhomologous contacts between chromosomes before homolog alignment has been attained. In htp-1 mutants, central region components prematurely associate with developing chromosome axes without regard for homolog pairing status. In the absence of both HTP-1 and HTP-2, synapsis is abolished, suggesting that the two proteins may have antagonistic functions in SC assembly or that both have different functions in promoting SC formation between homologs. Furthermore, HTP-1 is required to establish normal levels of recombination as assessed by RAD-51-marked recombination intermediates. These results support the emerging model that the HIM-3 family of proteins functions at the junction of essential meiotic processes to coordinate chromosome behavior with chromosome structure.

\section{Results}

htp-1 and htp-2 are paralogs of him-3

Sequencing of the C. elegans genome revealed three paralogs of him-3 (htp-1, htp-2, and htp-3), each encoding proteins that are marked by the presence of a HORMA domain, a structural feature thought to act as an adaptor for protein recruitment in proteins that recognize chro- 
matin states resulting from DNA adducts, DSBs, or nonattachment to the spindle (Aravind and Koonin 1998). $h t p-1$ and $h t p-2$ encode two highly homologous proteins of 352 amino acids that share $82 \%$ protein sequence identity with each other and $<30 \%$ protein sequence identity with HIM-3 and HTP-3. The homology between HTP-1 and HTP-2 is also reflected at the nucleotide sequence level in exon/intron structure and in promoter, terminator, and intron sequences; ClustalW alignment identifies an overall nucleotide identity of $84 \%$ over a stretch of 3618 bp that includes 2097 bp of $5^{\prime}$-untranslated sequence and $322 \mathrm{bp}$ of $3^{\prime}$-untranslated sequence (data not shown), suggesting that the two genes arose from a common ancestral gene. The genomes of the $C$. elegans relatives Caenorhabditis briggsae and Caenorhabditis remanei encode four and five HIM-3-like genes, respectively, of which two are clear HIM-3 and HTP-3 orthologs; however, the high level of homology between $h t p-1$ and $h t p-2$ makes the identification of their true orthologs among the remaining family members of the two species difficult.

\section{htp-1 and htp-2 function in meiotic chromosome} segregation

To investigate the function of $h t p-1$, we examined the phenotype associated with the predicted null allele gk174, a deletion that completely removes the coding sequence (see Materials and Methods). htp-1 mutant hermaphrodites segregate $94 \%$ inviable embryos; among those that survive, $24 \%$ develop into males (Table 1) (high incidence of males phenotype), indicative of a severe chromosome segregation defect (Hodgkin et al. 1979). Chromosome mis-segregation is often the result of defects occurring earlier in the meiotic cell cycle that interfere with recombination between homologous chromosomes, resulting in a decrease in chiasma formation. The events of meiotic prophase are particularly cytologically accessible in C. elegans hermaphrodite germlines, where a temporal succession of nuclei at different stages can be readily distinguished in DAPI-stained three-dimensionally preserved tissues (Francis et al. 1995). At the onset of meiosis, nuclei enter the transition zone of the germline (corresponding to the leptotene-zygotene stage) (Dernburg et al. 1998) and are distinguished by a crescent-shaped appearance resulting from the chromosome clustering that accompanies the initiation of homolog alignment. These are followed by pachytene-stage nuclei that are characterized by parallel DAPI-stained

Table 1. Segregation defects in the absence of htp-1 and htp-2

\begin{tabular}{lrc}
\hline Genotype & $\begin{array}{c}\text { \% embryonic } \\
\text { lethality }(n)\end{array}$ & \% males (95\% C.I.) \\
\hline$+/+$ & $2(3059)$ & $<0.3$ \\
$h t p-1 / h t p-1$ & $94.5(3223)$ & $23.8(19.5-28.3)$ \\
$h t p-/ h t p-1 ; h t p-2(R N A i)$ & $96.6(5442)$ & $33.3(29.8-36.7)$ \\
\hline
\end{tabular}

tracks corresponding to synapsed homologous chromosomes that are intimately connected along their entire lengths by the SC. Following desynapsis, six bivalents, corresponding to six pairs of homologs linked by chiasmata, are observed in nuclei at the last stage of meiotic prophase (diakinesis). In htp-1 mutant germlines, transition zone nuclei often show less polarization of the chromatin to one side of the nucleus, and the number of nuclei undergoing polarization of chromatin per germline is reduced compared with age-matched controls. The pachytene region of the germline is populated by nuclei in which the parallel DAPI-stained tracks indicative of the alignment of the chromosomes to a distance compatible with their close association by synapsis is readily observed (Fig. 3B, below). At the diakinesis stage, however, an average of 11.1 well-condensed bodies (range of 9-12; $n=37$ )-corresponding to the formation of up to three bivalents-is observed, indicating that htp-1 mutants are competent to form chiasmata but that HTP-1 is required to establish normal levels.

Since no htp-2 mutant exists, we used RNAi to examine the function of the gene; because the high level of sequence identity between $h t p-1$ and $h t p-2$ would result in cross-interference, the phenotype of $h t p-2(R N A i)$ was analyzed in the $h t p-1$ mutant background (Materials and Methods). htp-2(RNAi) does not target him-3 or htp-3 since both HIM-3 and HTP-3 localize normally in $h t p-1$; htp-2(RNAi) germlines (Fig. 2, below; data not shown). When both $h t p-1$ and $h t p-2$ are abolished, embryonic lethality remains at Htp-1 levels; however, the percentage of male progeny increases to $33 \%$, typical of mutants in which crossing over is abolished (Villeneuve 1994; Dernburg et al. 1998; Zalevsky et al. 1999; Zetka et al. 1999). Consistent with this observation, DAPI staining of $h t p$ 1; htp-2(RNAi) mutant germlines found that 12 univalents $(n=35)$ were consistently present in diakinesisstage nuclei, indicating that either HTP-1 or HTP-2 alone, or the two proteins functioning redundantly, are required for chiasma formation. Furthermore, the variably polarized phenotype of transition zone nuclei observed in htp-1 mutants becomes more dramatic and fewer nuclei with a more pronounced polarization were observed upon depletion of $h t p-2$ (data not shown). In the region of the germline that would normally correspond to the pachytene stage, a striking disorganization typical of mutants in which synapsis has failed to occur was observed (Zetka et al. 1999); no parallel alignment of chromosome pairs was detectable, and chromatin aggregates were evident at the periphery of the nuclei (Fig. 2, below).

\section{htp-1 mutants are defective in initial homolog alignment}

We observed that the loss of $h t p-1$ resulted in defects in the level of polarization of transition zone nuclei, a phenomenon associated with mutants that disrupt the process of homolog alignment at the onset of prophase (MacQueen and Villeneuve 2001; Couteau et al. 2004). To determine if $h t p-1$ is required for this process, we 
performed a time-course analysis of pairing using fluorescence in situ hybridization (FISH) and probes specific to several chromosomal loci on whole-mounted gonads (Fig. 1). During wild-type meiosis, homolog alignment initiates in the leptotene-zygotene stage (Dernburg et al. 1998 ) and is stabilized by synapsis during pachytene (MacQueen et al. 2002). In htp-1 mutant germlines, au-

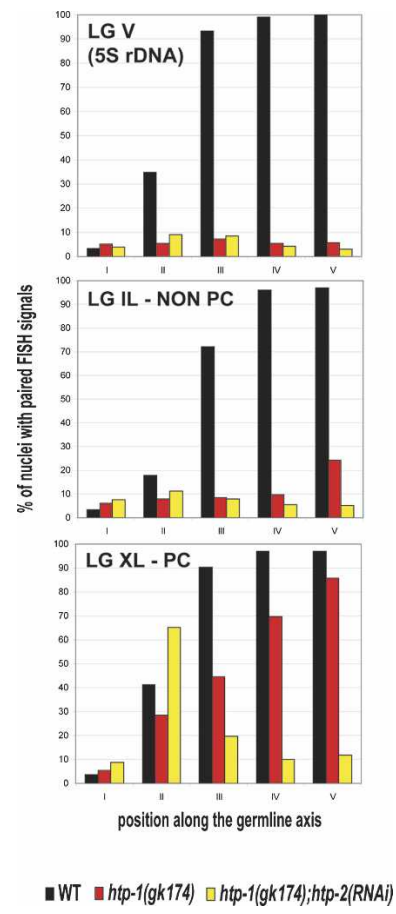

Figure 1. Time-course analysis of pairing levels in $h t p-1$ and htp-1; htp-2(RNAi) mutant germlines. Whole-mounted gonads were divided into five equivalently sized intervals along their distal-proximal axes (45-50 $\mu \mathrm{m}$ each) as in Couteau et al. (2004), each predominantly containing a population of nuclei corresponding to the following stages: (I) mitotic/premeiotic, (II) leptotene-zygotene, (III-V) early, mid-, and late pachytene. Singlecopy probes were used to monitor the pairing of the left ends of chromosomes I and X (LG-IL and LG-XL), and a 5S rDNA repetitive sequence probe was used to follow the pairing of the right arm of chromosome V (LG V). Histograms illustrate the level of pairing attained for each probe used in the genotypes tested. In wild type, homolog alignment initiated in the transition zone (zone II), and pairing levels rapidly increased and persisted throughout pachytene. (zones III-V). In $h t p-1$ and $h t p-1$; $h t p-2(R N A i)$ mutants, autosomal pairing levels were not different from the backgrounds levels observed in the mitotic/premeiotic region (zone I), except in late pachytene for LG IL in $h t p-1$ mutants $(p=0.002)$. A robust level of $\mathrm{X}$-chromosome pairing was observed in $h t p-1$ mutants, albeit at slightly reduced levels in comparison to wild type in zone II ( $p=0.0218)$, zone III, and zone IV $(p<0.0001)$. The alignment is, however, stabilized over time and finally reaches a level that is not statistically different from wild type in zone $\mathrm{V}(p=0.45)$. In $h t p-1$; $h t p-$ 2(RNAi) germlines, $\mathrm{X}$-chromosome pairing is at wild-type levels, but fails to persist and decreases over time to return to background levels in late pachytene (zone V). The numbers of scored nuclei and percentage of paired FISH signals are presented in Supplementary Table 1. tosomal pairing levels generally never rose above the background level detected in the mitotic/premeiotic region; however, a significant level of pairing was observed in late pachytene for the nonpairing center end of chromosome I (24\% paired FISH signals), indicating that while $h t p-1$ mutants are severely defective in the process of homolog alignment, the low level of alignment that is achieved is likely to be stabilized by synapsis. In contrast to the autosomes, the $\mathrm{X}$ chromosomes displayed a robust alignment activity; at the leptotene-zygotene stage, the number of paired FISH signals were $70 \%$ of wild-type levels, and during pachytene this level progressively increased to reach the wild-type level by late pachytene, suggesting that the process of X-chromosome homolog alignment may be slowed or delayed in htp-1 mutants, but is correctly stabilized via synapsis. Martinez-Perez and Villeneuve (2005) report substantially higher overall levels of autosomal pairing in htp-1 mutants (reaching a high of $33 \%$ for the 5 S rDNA probe), a difference that we attribute to variations in detection levels as a consequence of specimen fixation and probe generation. Furthermore, maternal age-related effects may also have contributed to the difference, since assessing the most severe $h t p-2$ loss-of-function phenotype required the dissection and processing of both $h t p-1$; $h t p-2(R N A i)$ and $h t p-1$ control worms 88 h post-L4 (rather than the usual $20 \mathrm{~h})$. Ultimately, the results of both studies are in agreement in that they demonstrate a requirement for HTP-1 in attaining wild-type levels of homolog alignment, and indicate a differential requirement for the protein in the process between autosomes and the sex chromosomes.

\section{$X$-chromosome alignment is not stabilized in htp-1; htp-2(RNAi) germlines}

Given the high level of X-chromosome alignment observed in $h t p-1$ mutants, we asked if early pairing of the sex chromosomes could be mediated by $h t p-2$. The depletion of $h t p-2$ by RNAi in $h t p-1$ mutants did not eliminate the initial alignment of the $\mathrm{X}$ chromosomes (Fig. 1), suggesting that X-chromosome alignment does not require HTP-1 or HTP-2. However, in the absence of both proteins, the successful initial alignment of the $\mathrm{X}$ chromosomes was not accompanied in later stages by its stabilization, since alignment was progressively lost to return to background levels in late pachytene. Furthermore, we observed that the autosomal pairing detected for the left end of chromosome I in late pachytene nuclei of $h t p-1$ mutants alone was also reduced to background levels in $h t p-1$; $h t p-2(R N A i)$ mutant germlines. This failure to stabilize early homolog alignment, coupled with the lack of parallel DAPI-stained tracts typical of synapsed chromosomes in the pachytene region of $h t p-1$; htp-2(RNAi) germlines, and the absence of chiasmata at diakinesis are diagnostic of mutants specifically defective in SC assembly (MacQueen et al. 2002; Colaiácovo et al. 2003), indicating that $h t p-2$ alone, or $h t p-1$ and $h t p-2$ functioning redundantly, are required for the stabilization conferred by synapsis during pachytene. 
htp-1 is required to prevent synapsis between nonhomologous chromosomes

Despite the severe defect in homolog alignment in htp-1 mutants, the pachytene-stage nuclei reveal the extensive presence of the DAPI-stained parallel tracks typical of synapsed chromosomes. To investigate Htp-1 chromosome structure during pachytene, we used HIM-3 as a marker for chromosome axes (Zetka et al. 1999) and SYP1 , a structural component of the SC central region (MacQueen et al. 2002), as a marker of SC formation (Fig. 2). Previous studies have shown that HIM-3 localizes to the cores of both synapsed and unsynapsed chromosomes and is required for synapsis and for SYP-1 localization (Zetka et al. 1999; Couteau et al. 2004). In wild-type pachytene nuclei where chromosomes are fully aligned and synapsed along their lengths, SYP-1 stretches colocalized with HIM-3 on the DAPI-stained tracks. The pachytene nuclei of $h t p-1$ mutants show a wild-type localization of HIM-3 and also exhibit extensive colocalization of HIM-3 and SYP-1 on parallel chromosome cores, indicating that the majority of chromosomes are engaging in synapsis. However, stretches of HIM-3 tracks corresponding to isolated single DAPI-stained

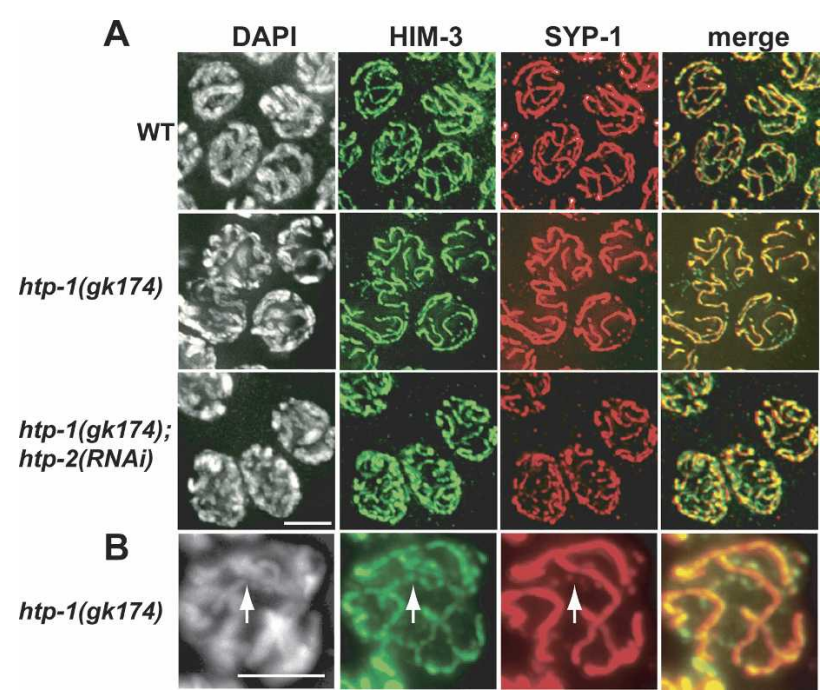

Figure 2. Defective synapsis in $h t p-1$ and $h t p-1$; $h t p-2$ (RNAi) mutants. (A) In wild-type germlines, parallel DAPI-stained tracts corresponding to two synapsed chromosome axes show precise colocalization of the meiotic chromosome axis component HIM-3 and the SC component SYP-1 in mid-pachytene nuclei. In $h t p-1$ mutants, DAPI-stained chromosomes are aligned to a distance compatible with synapsis (inset) and HIM-3 and SYP-1 colocalize, suggesting the presence of SC. In htp-1; htp-2(RNAi) depleted nuclei, HIM-3 and SYP-1 extensively localize to chromosomes; however, nuclei are disorganized in appearance; no alignment of DAPI-stained chromosomes is evident, and chromatin masses are dispersed throughout the nucleus. (B) Squashed preparations of pachytene nuclei of $h t p-1$ mutants reveal the presence of unsynapsed axes; arrows indicate a single unaligned DAPI-stained stretch, corresponding to an unsynapsed chromosome segment. HIM-3 is localized to the unsynapsed core, and SYP-1 is detectable as a discontinuous and thin thread that follows the HIM-3 pattern. Bars, $4 \mu \mathrm{m}$. tracks are also evident, indicating the presence of stretches of unsynapsed axes. Furthermore, a low level of SYP-1 association to these unsynapsed cores can often be detected. Thus, while chromosomes engage in extensive synapsis in $h t p-1$ mutants, as evidenced by the presence of aligned DAPI-stained tracks and by SYP-1 staining between these aligned axes, this synapsis does not reflect the stabilization of homologous chromosome alignment given the low level of pairing detected by FISH. In this context, the detection of unsynapsed stretches of axes is likely the result of structural constraints imposed by synapsis between imprecisely aligned nonhomologous chromosome cores. Our results demonstrate that while HTP-1 is required to attain normal levels of homolog alignment, failure to do so in the absence of the protein results in robust and inappropriate synapsis between nonhomologous chromosomes, indicating that HTP-1 is required to prevent the stabilization of inappropriate contacts through synapsis.

Precocious loading of SC components to chromosome axes in htp-1 mutants

The extensive nonhomologous synapsis observed in htp-1 mutants raised the possibility that the defect in homolog alignment could originate in a defect in the coordination of alignment with synapsis; inappropriately early initiation of synapsis between chromosomes could entrench nonhomologous contacts and interfere with the process of homolog alignment. To investigate this possibility, we took advantage of the robust, but delayed alignment observed for the $\mathrm{X}$ chromosomes in htp-1 mutants to examine the coordination of homolog alignment and SC component loading in a time-course analysis of the two processes in transition zone nuclei. Using SYP-1 recruitment to developing chromosome axes as a marker for an early step in synapsis initiation, we determined the incidence of paired X-chromosome FISH signals associated with a stretch of SYP-1 staining on the same DAPI tract (Fig. 3). During wild-type meiosis, leptotenezygotene nuclei formed three distinct populations that reflect the early and orderly coordination of homolog alignment with synapsis: nuclei with unpaired FISH signals without an associated tract of SYP-1 (49\%), paired FISH signals without an associated tract of SYP-1 (27\%), and paired FISH signals on a tract of SYP-1 (24\%). The high proportion of nuclei showing paired FISH signals without SYP-1 association suggests that local homolog alignment is not immediately rewarded with synapsis, but that stabilization may be initiated once a threshold level of alignment along the length of the chromosome has been achieved. In early pachytene, the number of nuclei exhibiting both stabilization of homolog alignment and SYP-1 tracts on the X chromosome rapidly increases to $93 \%$, revealing the near perfect coordination of the processes of homolog alignment and synapsis. A low level of the unexpected class of SYP-1 tracts associated with one or both unpaired chromosomes was also observed and is likely the consequence of background error for our detection level. 


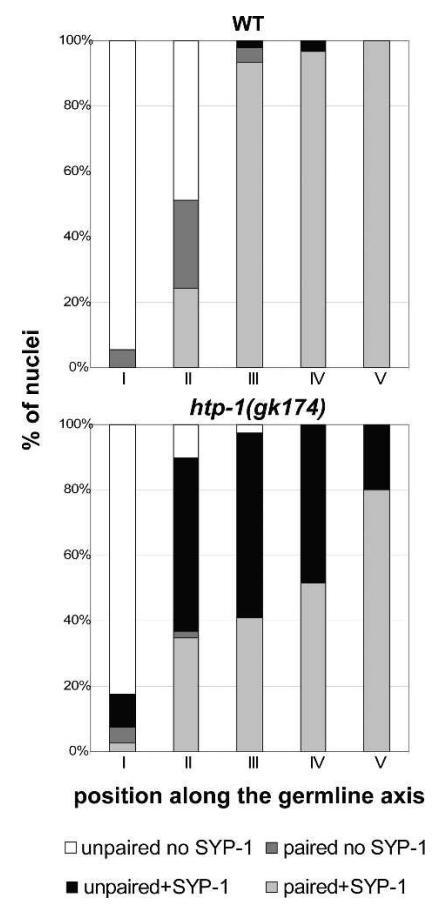

Figure 3. SYP-1 loading is uncoupled from X-chromosome pairing in $h t p-1$ mutants. Time-course analysis of X-chromosome pairing and SYP-1 recruitment on whole-mounted, threedimensionally preserved gonads of wild-type controls and $h t p-1$ mutants. For scoring, germlines were divided into five equivalently sized zones as described in Figure 1. For each nucleus, the status of X-chromosome alignment was first ascertained (paired or unpaired FISH signals), and then the DAPI-stained chromosome region to which the FISH signal mapped was examined for SYP-1 colocalization. In the wild-type germlines, two predominant classes of nuclei are observed: those with unpaired FISH signals, neither of which was associated with detectable SYP-1 tracts, and those with a paired FISH signals associated with an SYP-1 tract. In htp-1 mutant germlines, a third category of nuclei appears at a high frequency: those in which FISH signals are unpaired, but at least one of them lies on a tract of SYP-1. The numbers of scored nuclei in each category are presented in Supplementary Table 2.

In striking contrast, we observed an inappropriately early association of SYP-1 with chromosomes irrespective of their pairing status in the leptotene-zygotene nuclei of $h t p-1$ mutant germlines. While only $24 \%$ of wildtype nuclei of the transition zone showed SYP-1 association with chromosomes, $87 \%$ of Htp-1 nuclei of the same stage already showed SYP-1 tracts, indicating that SYP-1 loading occurs prematurely in the absence of HTP-1. Importantly, in more than half of these nuclei, the SYP-1 tract was associated with unpaired FISH signals, a class not observed in wild type. This pattern was maintained into early pachytene, where the total level of SYP-1 association with the X chromosome (97\%) approximates that observed in wild type $(93 \%)$, although in more than half of the nuclei $(56 \%)$ the chromosomes are not homologously aligned. These results demonstrate a robust and premature loading of SYP-1 loading to mei- otic chromosomes in $h t p-1$ mutants that is uncoupled from the progression of X-chromosome alignment.

\section{SYP-1 localizes to immature chromosome axes in htp-1 mutants}

In $h t p-1$ mutants, we observed the inappropriately early association of the central element protein SYP-1 with chromosomes still engaged in the process of homolog alignment, suggesting that HTP-1 functions in preventing the loading of SC components before homolog alignment is attained. Because HIM-3 is required at chromosome axes for homolog alignment to occur, we investigated the recruitment of HIM-3 to developing axes in $h t p-1$ mutants relative to the loading of the central element components SYP-1 and SYP-2 /Colaiácovo et al. 2003). During wild-type meiosis, HIM-3 localization assumes three general patterns that reflect the progression of chromosome axis formation; a weak and diffuse localization in the more proximal nuclei of the premeiotic zone nuclei; foci and stretches that overlap with DAPI staining in leptotene-zygotene nuclei; and contiguous localization coincident with the six synapsed chromosomes at pachytene (Zetka et al. 1999). In wild-type germlines, SYP-1 is dependent on HIM-3 for association to chromosomes (Couteau et al. 2004) and first appears as aggregates and bright stretches in the same leptotenezygotene nuclei in which HIM-3-marked axes are assembling (MacQueen et al. 2002); SYP-1 localizes to both stretches of HIM-3 and to a subset of HIM-3 foci, but is always less abundant than HIM-3 at chromosome axes at this stage (Fig. 2). We first determined if the early association of SYP-1 localization observed in htp-1 mutants was still dependent on prior recruitment of HIM-3. In the absence of HIM-3, the precocious association of both SYP-1 (Fig. 4) and SYP-2 (data not shown) to chromosome cores during leptotene-zygotene in htp-1 mutants was abrogated, and both proteins localized to nuclear aggregates, indicating that their loading in $h t p-1$ mutants remains dependent on HIM-3. In htp-1 mutants, as in wild type, HIM-3 first appeared as bright foci in leptotene-zygotene nuclei, suggesting that no detectable delay occurred in the loading of HIM-3. However, fewer contiguous stretches of HIM-3 formed in these nuclei, while the relative staining of SYP-1 was much brighter than observed for wild-type nuclei of the same stage. Noticeably, robust tracts of SYP-1 were observed on axes where HIM-3 was not yet present in stretches, suggesting the inappropriate aggregation/polymerization of SYP-1 tracts relative to axis formation. Since the early association of SYP-1 (and SYP-2) (data not shown) could be the result of a defect in axis formation per se, the process was examined in the transition zone nuclei of htp-1 mutants by monitoring the localization of REC-8, a chromosome core component required for HIM-3 recruitment during meiosis (Pasierbek et al. 2001). REC-8 localized without delay, at appropriate levels, and with normal kinetics in transition zone nuclei of htp-1 mutants, suggesting that the decrease in HIM-3 localization 


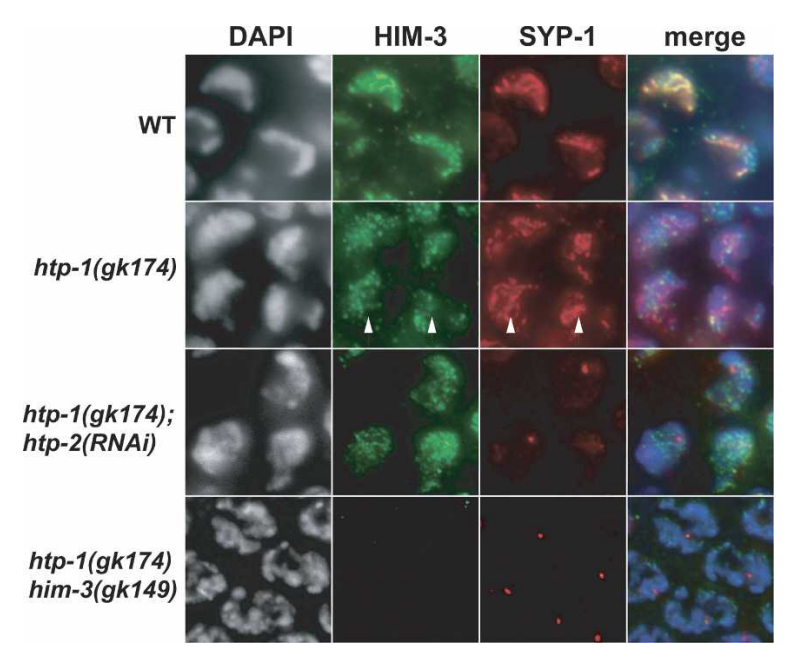

Figure 4. Coordination of axis formation with SYP-1 recruitment. HIM-3 and SYP-1 localization in leptotene-zygotene nuclei of whole-mounted gonads. Nuclei corresponding to the leptotene-zygotene stage (transition zone) were identified in wildtype, $h t p-1$, and $h t p-1$; $h t p-2(R N A i)$ mutant germlines by position in the gonad (zone II) and by the clustering of the DAPIstained chromatin to one side of the nucleus. Similar to him3(gk149)-null mutants (Couteau et al. 2004), no chromosome clustering is observed in htp-1(gk174) him-3(gk149) double mutants, and zone II nuclei expressing SYP-1 are shown. In wildtype germlines, HIM-3 localizes to developing chromosome cores, and SYP-1 localizes to a subset of HIM-3 stretches. A similar level and pattern of HIM-3 localization is present in htp-1 mutants; however, SYP-1 staining is generally more abundant relative to HIM-3 staining, and contiguous SYP-1 stretches can already be detected at chromosome axes where HIM-3 localization is still punctate (arrows). In both $h t p-1$; $h t p-2$ (RNAi) and htp-1 him-3, mutants, SYP-1 fails to localize to chromosomes and instead appears as the single nuclear aggregate observed in him-3 mutants in which no synapsis occurs (Couteau et al. 2004). Bars, $4 \mu \mathrm{m}$.

observed in these nuclei is not the result of a gross defect in axis formation.

\section{Chromosomes fail to synapse in htp-1; htp-2(RNAi) mutant germlines}

Our results demonstrate that HTP-1 and HTP-2 acting redundantly, or HTP-2 alone, are required for the assembly of the SC, a function that could be structural or regulatory in nature. To investigate the role of the proteins in synapsis, chromosome axis structure and the behavior of SC components were investigated in $h t p-1$; $h t p-2(R N A i)$ mutant germlines. HIM-3 appeared on time and at wildtype levels throughout prophase, suggesting that chromosome core formation proceeded normally in the absence of both $h t p-1$ and $h t p-2$. At pachytene, SYP-1 loaded extensively onto chromosome cores and colocalized with HIM-3, despite the absence of any evidence of synapsis (see above), a localization pattern that has also been observed for SYP-1 in another asynaptic mutant, syp-3 (M. Colaiácovo, pers. comm.). In contrast to htp-1 mutants alone, however, no SYP-1 (or SYP-2) (data not shown) association with axes was detected in leptotenezygotene nuclei upon depletion of $h t p-2$ in the $h t p-1 \mathrm{mu}-$ tant background, and the protein localized to the nuclear aggregate (Fig. 2) observed in him-3 mutants in which chromosomes fail to synapse. Thus, while HTP-1 functions in preventing the inappropriate association of central element proteins with chromosomes, HTP-1 and HTP-2 are redundantly required, or possibly HTP-2 alone is required for their loading specifically at the leptotenezygotene stage, suggesting that the two genes have antagonistic functions in the control of SC component loading during early meiotic prophase or that their functions are distinct, but required for normal SC formation between homologs. In either case, the localization of SYP-1/2 to nuclear aggregates in the absence of HTP-1 and HTP-2 is consistent with a defect in the loading of these central region components, similar to that observed in axis-defective him-3 mutants (Couteau et al. 2004).

\section{Crossing over is severely impaired in htp-1 mutants}

Although we observed a lack of coordination between SYP-1 loading and X-chromosome homolog alignment during early prophase in htp-1 mutants, this alignment was ultimately stabilized to wild-type levels by late pachytene, suggesting the formation of SC between the $\mathrm{X}$ chromosomes. Given the central role of the SC in crossover formation in most systems, we investigated the competency of the SC formed in $h t p-1$ mutants to support crossing over between the homologously-aligned $\mathrm{X}$ chromosomes. The frequency of crossing over of a large interval on the left arm of the X chromosome was reduced to $25 \%$ of the wild-type frequency (Table 2), demonstrating that $h t p-1$ mutants are competent for crossover formation, but at severely reduced levels. Furthermore, the presence of the occasional diakinesis nucleus with up to three bivalents in $h t p-1$ mutants suggests that crossing over can also occur on the autosomes, presumably in regions that have attained homologous synapsis.

htp-1 mutants are defective in the formation of RAD-5- marked early recombination intermediates

To investigate the origin of the recombination defect in htp-1 mutants, we first assessed the formation of early recombination intermediates by monitoring the localization of RAD-51, the single nematode representative of

Table 2. X-chromosome crossing over is severely reduced in htp-1 mutants

\begin{tabular}{lcccc}
\hline Genotype & WT & Dpy & Unc & cM (95\% C.I.) \\
\hline $\begin{array}{l}\text { dpy-3 unc-3/++ } \\
\text { dpy-3 unc-3/++; }\end{array}$ & 873 & 229 & 237 & $39.3(37.4-41.2)$ \\
htp-1/htp-1 & 716 & 38 & 58 & $9.9(6.8-12.4)$ \\
\hline
\end{tabular}


the highly conserved recA protein family required for early events in the repair of meiotic DSBs, the initiating events of meiotic recombination. Since RAD-51 localization is dependent on the formation of DSBs (Colaiácovo et al. 2003), anti-RAD-51 antibodies were used to evaluate the level of DSB formation and to monitor the progression of meiotic recombination in a time-course analysis on whole-mounted gonads (Fig. 5). Consistent with previous observations, in wild-type germlines, the number of RAD-51 foci peaked in zone III, where $76 \%$ of the nuclei have a least one RAD-51 focus (Couteau et al. 2004). In the germlines of $h t p-1$ mutants, the peak level of RAD-51 foci also appeared in zone III; however, only $4.6 \%$ of the nuclei in this zone were positive for RAD- 51 foci, and no more than one focus was observed per nucleus. Thus, while an average of 257 foci per 100 nuclei can be detected in zone III of wild-type germlines, an average of only 4.6 foci per 100 nuclei were detected in the same zone in $h t p-1$ mutants, corresponding to a RAD-51 loading level only $1.8 \%$ of that observed in wildtype germlines. Martinez-Perez and Villeneuve (2005) report substantially higher detection of RAD-51 foci in htp-1 mutants, a discrepancy that we attribute to a higher background in our specimens as a consequence of our use of an antibody against the nucleolar marker fi-

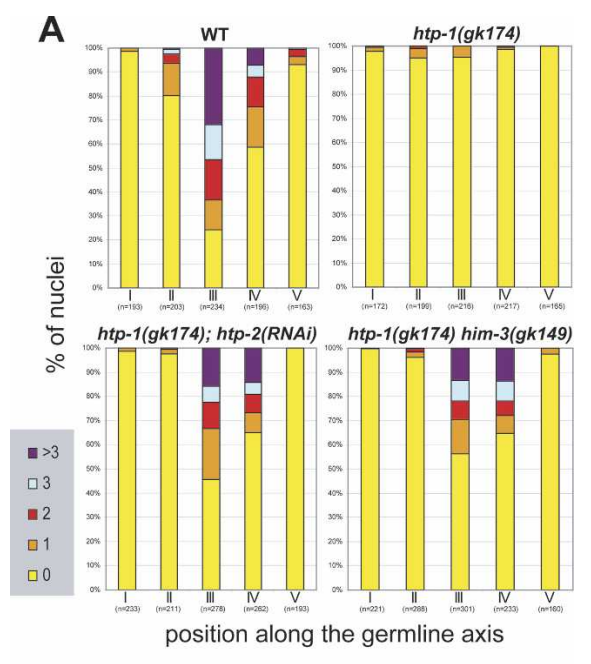

B

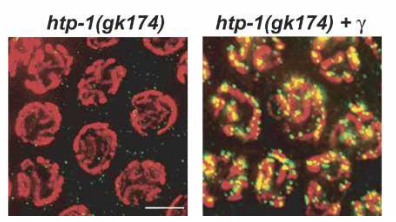

Figure 5. Formation of RAD-51-marked recombination intermediates increases in asynaptic htp-1; him-3 mutants and in $h t p-1 ;$ t $t p-2(R N A i)$. (A) Histograms showing the quantitation of RAD-51 foci from germlines of animals of the indicated genotypes. Nuclei were classified into five categories depending on the number of RAD-51 foci that were scored; category $>3$ includes nuclei showing 4-11 RAD-51 foci, but the vast majority of the nuclei in this category show 4-6 RAD-51 foci/nucleus in every genetic background tested. (B) Early pachytene nuclei of control and $\gamma$-irradiated $h t p-1$ mutant germlines stained with DAPI (red) and $\alpha$-RAD-51 (green). Bars, $4 \mu \mathrm{m}$. brillarin as a positive control for staining conditions in our RAD-51 experiments (Materials and Methods). However, our results are within the range of previous reports (Couteau et al. 2004) and support the conclusion arrived at by both groups; that $h t p-1$ mutants show a deficit of RAD-51-marked recombination intermediates.

To determine if the severe reduction in RAD-51 focus formation observed in the htp-1 mutant background could originate in a defect in the ability to load RAD-51, we tested if RAD-51 focus formation increased in the presence of artificial $\gamma$-irradiation-induced DSBs, previously shown to be able to support meiotic recombination initiation (Dernburg et al. 1998; Kelly et al. 2000). Irradiated $h t p-1$ germline nuclei showed a dramatic increase in RAD-51 focus formation (Fig. 5) that is similar to the level observed in irradiated wild-type controls (data not shown), demonstrating that $h t p-1$ mutants are competent for RAD-51 loading in the presence of artificially introduced DSBs.

Formation of early recombination intermediates increases in asynaptic htp-1 him-3 mutants and in htp-1; htp-2(RNAi)

Although the precise timing and kinetics of DSB formation are not known in C. elegans, it is likely that the majority of events are initiated before early pachytene, when the peak level of RAD-51-marked early recombination events are detected. Since central element/lateral element components have been intimately tied to the regulation of early recombination events in yeast (Schwacha and Kleckner 1997), flies (Jang et al. 2003), and worms (Jantsch et al. 2004), the premature association of these synapsis-associated proteins with chromosome axes during early prophase and the severe reduction in RAD-51-marked recombination intermediates observed in htp-1 mutants raised the possibility that these two defects are linked. We examined RAD-51 levels in htp-1 him-3 and htp-1; htp-2(RNAi) double mutants in which lateral/central element proteins dependent on HIM-3 or HTP-1/HTP-2 function for loading to chromosome axes are unable to do so. RAD-51 foci appear on time and with wild-type kinetics in him-3-null mutants in which chromosomes fail to align or synapse, indicating that stable homolog pairing is not a prerequisite to recombination initiation or progression per se (Couteau et al. 2004). In htp-1 him-3 mutant germlines, the level of RAD-51 foci peaked in zone III to a level 23-fold higher than observed in $h t p-1$ mutants alone (108 foci/100 nuclei vs. 4.6 foci/100 nuclei, respectively) (Fig. 5). Similar to the effect of the him-3 mutation, depletion of $h t p-2$ in the $h t p-1$ mutant background also markedly restored the level of RAD-51 foci, resulting in a 12-fold increase in the peak of RAD-51 foci (54.3 foci/100 nuclei). In both $h t p-1$ him-3 and $h t p-1$; $h t p-2$ (RNAi) mutant germlines, RAD-51 foci disappeared with wild-type kinetics (no foci detectable in the late pachytene region), indicative of a full ability to repair DSBs on time. Our results demonstrate a dramatic increase in the levels of RAD-51-marked recombination events in $h t p-1$ mutants 
when SC central/lateral element components requiring HIM-3 or HTP-1/2 for association to chromosome axes during leptotene-zygotene are prevented from doing so. This result is consistent with our interpretation that $h t p-1$ functions in preventing the association of these components in the context of chromosome axes at this stage and that this association can interfere with early events in recombination.

\section{Discussion}

HTP-1 and HTP-2 have meiotic functions in synapsis and recombination

Our analysis has found that both $h t p-1$ and $h t p-2$ have essential roles during meiosis at the junction of chromosome alignment, synapsis, and recombination. In the absence of HTP-1, chromosomes are proficient to undergo extensive synapsis; however, synapsis occurs almost exclusively between nonhomologous chromosomes, demonstrating that HTP-1 functions in preventing the stabilization of inappropriate contacts through synapsis-a role that has been attributed to a very limited number of genes among all the species studied to date. Although positioned between nonhomologous partners, several lines of evidence suggest that the SC formed in $h t p-1$ mutants may be wild type in structure; mutants are competent for recombination and chiasma formation (albeit at drastically reduced levels), X-chromosome alignment is appropriately stabilized, and chromosomes are paired at pachytene at a distance compatible with the presence of a SC. In htp-1; htp-2(RNAi) germlines, cytological defects consistent with essential functions for the proteins in SC assembly per se are observed; the failure to load SYP-1 to chromosomes at leptotene-zygotene, a lack of parallel DAPI-stained tracks coupled with a failure to stabilize homolog alignment at pachytene, and the failure to form chiasmata at diakinesis (Colaiácovo et al. 2003; Couteau et al. 2004). That SYP-1 localization during early prophase is restricted to the polycomplex-like aggregates observed in other SC-defective mutants in the absence of both $h t p-1$ and $h t p-2$, but is localized extensively to chromosome cores at pachytene, suggests that HTP-1 and HTP-2 are redundantly required or possibly HTP-2 alone is required to promote central region component association to chromosomes specifically at leptotene-zygotene when synapsis is being initiated. Alternatively, the two proteins may have antagonistic functions in synapsis, with HTP-1 inhibiting and HTP-2 promoting SC assembly. Although HTP-1 and HTP-2 are highly homologous, the HORMA domain-predicted to facilitate protein-protein interactions (Nasmyth 2005) encompasses almost the entire predicted protein sequence of both proteins, and even subtle changes in the motif may result in changes in protein interaction preference that result in their participation in opposing pathways. Furthermore, our results suggest that while HTP-1 is required for establishing normal levels of recombination initiation, both HTP-1 and HTP-2 are required for chiasma formation per se.

\section{HTP-1 coordinates homolog alignment with synapsis}

During wild-type meiosis, only homologous chromosome alignment is rewarded with synapsis, despite the ready availability of SC components in meiotic nuclei. In $h t p-1$ mutants, the essential temporal coordination of the two processes is uncoupled, indicating the existence of a regulatory mechanism under genetic control. In addition to the extensive nonhomologous synapsis observed at pachytene, $h t p-1$ mutants show severe defects in homolog alignment during early prophase. Although we cannot exclude the possibility that HTP-1 functions directly in the process of homolog alignment, the fact that the alignment of the X chromosomes in htp-1-null mutants progresses efficiently to reach wild-type levels eliminates a general role for the protein in the process. More importantly, we observed that SYP-1 loading onto chromosomes occurred precociously and at inappropriately high levels relative to the process of homolog alignment and the level of HIM-3 at developing chromosome axes, suggesting that the primary defect is the inappropriate loading of SC components. SYP-1 could also be detected at unsynapsed chromosome cores at pachytene in $h t p-1$ mutants, indicating that the protein can associate with individual chromosome axes, and SYP-2, a second putative TF component, comislocalizes with SYP-1 in htp-1 mutants (data not shown), indicating that SYP-1 is not the only SC component that is deregulated. However, the fact that pairing levels are not restored in $h t p-1$; syp-2 double mutants (Martinez-Perez and Villeneuve 2005), indicates that the pairing defect observed in htp-1 mutants cannot solely be the result of the premature association of these two central element components with chromosome axes. Instead, we propose that in addition to SYP-1/SYP-2, lateral element components that respond to the same signals for SC assembly are also deregulated in the absence of HTP-1; these components would be predicted to associate with developing chromosome axes in syp-1/syp-2 mutants and could disrupt the chromosome axis morphogenesis required for proper homolog alignment. We favor the hypothesis that a role of HTP-1 is to globally prevent the association of components required for SC assembly (both axis-associated lateral element components and central region components) to chromosomes undergoing homolog alignment at the leptotene-zygotene stage until conditions to assemble SC between homologous chromosomes have been met. In this context, HTP-1 may function to either prevent SC components from assembling between nonhomologs or to control the timing at which chromosomes can accumulate these components. Thus, HTP-1 is required to coordinate homolog alignment with its stabilization by synapsis, indicating the existence of a mechanism functioning in early prophase to monitor the status of homolog alignment and couple it to SC formation.

An outstanding question is why the inappropriately early loading of lateral/central element components relative to chromosome pairing might result in severe pairing defects and nonhomologous synapsis on the auto- 
somes, yet the $\mathrm{X}$ chromosomes are able to attain high levels of stable homolog alignment. One possibility is that stabilization of X-chromosome alignment invokes $\mathrm{X}$-specific mechanisms that are less dependent on known SC components essential for autosomal stabilization of alignment. In non-null him-3(me80) mutants, for example, reduced levels of HIM-3 associate with chromosome axes; because HIM-3 is required for synapsis, this results in a drastic reduction in homologous synapsis on the autosomes, however, X-chromosome alignment is stabilized to near wild-type levels (Couteau et al. 2004), and extensive contiguous SC capable of supporting chiasma formation is assembled despite the defect in axis formation (Nabeshima et al. 2004). Therefore, it is possible that the premature loading of SC components in htp-1 mutants interferes with HIM-3-mediated homolog alignment on the autosomes, resulting in nonhomologous synapsis, but that alignment and its subsequent stabilization proceed efficiently on the $\mathrm{X}$ chromosome, despite the reduction in HIM-3 function.

\section{The role of HTP-1 in recombination}

In this study we have shown that $h t p-1$ mutants are defective in the recombination process; mutants exhibit a drastically reduced level of RAD-51 foci throughout early prophase and a deficit of chiasma at diakinesis. Is HTP-1 a component of the recombination machinery? The lack of homology between HTP-1 and the components of the recombination machinery, renowned for their high level of evolutionary conservation, argues against a role for HTP-1 as a core component in this process; however, the possibility that it plays an undescribed function in the process cannot be eliminated.

In maize phs1 mutants, nonhomologous chromosome synapsis is also accompanied by a drastic reduction in RAD-51 foci, despite evidence for extensive DSB formation, suggesting that the protein may be required for RAD-51 loading (Pawlowski et al. 2004). A direct role for HTP-1 in RAD-51 recruitment, or in its expression, is unlikely given that $h t p-1$ mutant germlines show extensive RAD-51 loading when artificial DSBs are introduced, demonstrating that $h t p-1$ mutants are competent for higher levels of RAD-51 focus formation. Instead, an explanation may lie in a role for HTP-1 in establishing a normal level of DSBs and/or the barrier to using the sister chromatid as a repair template; in the absence of the barrier, a more rapid turnover of recombination intermediates would also result in a deficit of RAD-51-marked foci in $h t p-1$ mutants. Our results are consistent with those of Martinez-Perez and Villeneuve (2005), in identifying a function for HTP-1 at both levels of the recombination process. First, we observe a drastic overall decrease in RAD-51 foci formation that is not observed in mutants in which recombination initiation proceeds normally, but in which the barrier to repairing recombination intermediates using the sister chromatid as a template is defective (Couteau et al. 2004). Second, the delay in the progression of RAD-51-marked intermediates observed in asynaptic syp-2 mutants (Colaiácovo et al. 2003) is alleviated in $h t p-1 ; h t p-2(R N A i)$ germlines in which chromosomes also fail to synapse, consistent with the interpretation that $h t p-1$ is required to establish the barrier.

In our study we observed that the levels of RAD-51 foci increased in htp-1; htp-2(RNAi) and htp-1 him-3 mutant germlines in which chromosomes fail to synapse and the precocious loading of the central element components SYP-1 and SYP- 2 is abrogated. Since $h t p-1$; syp-2 mutants show no rescue of the RAD-51-defective phenotype (Martinez-Perez and Villeneuve 2005), this defect cannot be attributed to the precocious association of SYP-1 and SYP-2. Instead, we propose that some of the deficit of RAD-51 foci observed in $h t p-1$ mutants may have the same origin as the defects in homolog alignment; the association of lateral element and/or other central region components with chromosome axes abnormally early in the meiotic program may also disrupt early steps in the recombination process. In this scenario, like SYP-1/SYP-2, a subset of these components requires HIM-3 and HTP-2 to associate with chromosome axes and is competent to interfere with early steps in the recombination process in $h t p-1$ mutants; the $z h p-3$ protein, for example, localizes to the SC in a HIM-3 and synapsis-dependent manner and is required for the processing of DSBs into crossovers (Jantsch et al. 2004). The precocious localization of such LE/CE components to chromosomes in $h t p-1$ mutants could signal that synapsis has been initiated and that progress through prophase is more advanced than it is, resulting in a narrower window for recombination initiation, or could directly engage chromatin, similar to the behavior of some polycomplexes (Bhuiyan et al. 2003), and disrupt conditions for DSB formation and/or the processing of DSBs into crossovers.

\section{Conclusions}

We have identified HTP-1 as a key regulator of synapsis that is required to prevent premature assembly of SC components relative to homolog alignment and chromosome morphogenesis at the onset of meiosis. This function is remarkably important, since SC proteins have been shown in many examples to be able to polymerize spontaneously and without regard for DNA homology, resulting in nonhomologous synapsis and ultimately in chromosome mis-segregation. Our study suggests that premature SC assembly onto immature chromosome axes can interfere with the process of homolog alignment and recombination initiation. Furthermore, we identify an essential function for HTP-1 and HTP-2 functioning redundantly, or HTP-2 alone, in an early step of SC formation. We suggest that HTP-1 and HTP-2 may have opposing roles in SC initiation: one by inhibiting SC component recruitment to chromosomes before homologs are aligned and the second by licensing their loading. Lastly, our study indicates that the HIM-3 family of proteins has essential functions in the coordination of early meiotic events. 


\section{Materials and methods}

\section{Genetics}

C. elegans strains were cultured following the methods described by Brenner (1974), and all experiments were conducted at $20^{\circ} \mathrm{C}$. The wild-type $\mathrm{N} 2$ strain var. Bristol and the mutations used in this study were obtained from the Caenorhabditis Genetic Center (University of Minnesota, St. Paul) or from the $C$. elegans Gene Knockout Consortium. The htp-1(gk174) allele was used for all experiments described in this study, and the mutant strain was backcrossed three times to N2 before analysis; $g k 174$ is a 1462 -bp deletion that covers the entire predicted coding sequence including $203 \mathrm{bp}$ upstream of the ATG start codon and 59 bp downstream of its translation termination signal.

The following mutations and rearrangements were used: LG IV, him-3(gk149); htp-1(gk174) LG X, unc-1(e719), dpy-3(e27).

Recombination frequencies were measured in hermaphrodites as described in Zetka et al. (1999) using the statistics of Crow and Gardner (1959).

\section{RNA interference}

To investigate the function of $h t p-2, \mathrm{RNA}$ interference experiments were performed as previously described (Fire et al. 1998). $h t p-2$ dsRNA was produced under standard conditions using the cDNA yk458g2 as template for in vitro transcription with T3 and T7 RNA polymerases (Invitrogen), purified using mini-columns (QIAGEN), and resuspended for injection at a concentration of $0.5-1 \mu \mathrm{g} / \mu \mathrm{L}$. All injections were performed on 20 -h postL4 or 24-28-h post-L3 hermaphrodites, and cytological analyses were performed 68-72 h post-injection. The gonads of agematched wild-type or $h t p-1$ worms were then microdissected, fixed, and processed in parallel, as described below. The number of chromosomes in diakinesis nuclei in both $h t p-1$ mutants and in $h t p-1$; $h t p-2(R N A i)$ germlines was determined by counting the number of DAPI-stained bodies in different focal planes. Because the high degree of homology between $h t p-1$ and $h t p-2$ makes specific targeting of $h t p-2$ through RNAi unfeasible, the phenotype of $h t p-2$ was assessed in the $h t p-1$ mutant background. The efficacy of the RNAi was demonstrated by the fact that injected worms consistently showed a phenotype distinct from htp-1 mutants, namely, (1) the absence of parallel tracks of chromosomes at pachytene after DAPI staining, and (2) the consistent presence of 12 univalents at diakinesis (i.e., the absence of any bivalents). Furthermore, the progeny of the injected mothers showed a significant increase in the frequency of male progeny (see Results).

\section{Fluorescence in situ hybridization and immunostainings}

Labeled probe generation for FISH analysis, the quantitation of homologous chromosome alignment, and antibody staining procedures were performed as described in Couteau et al. (2004). The $5 \mathrm{~S}$ rDNA probe was generated by PCR amplification (Dernburg et al. 1998), and the X left and I left probes were produced using the following mixture of cosmids and YACs (Couteau et al. 2004): B0554, R11G11, ZK6 (I left); Y51E2, C31G9, M02E1, $\mathrm{B} 0310$ (X left). The following primary antibodies and dilutions were used: rabbit anti-HIM-3 (1:200) (Zetka et al. 1999), guinea pig anti-SYP-1 (1:200) (MacQueen et al. 2002); rabbit antiCeRAD51 (1:100) (Colaiácovo et al. 2003); and mouse monoclonal anti-fibrillarin (1:200; Encor Biotechnology). Two different primary antibodies were used in each immunostaining experiment to control for antibody penetration and fixation. When
RAD-51 antibody was used, anti-fibrillarin was used as a positive control of the staining conditions. The following secondary antibodies (and dilutions) were used: Alexa Fluor 555 goat antiguinea pig (1:500; Molecular Probes); Alexa Fluor 488 goat antirabbit (1:500; Molecular Probes); and Cy3-conjugated donkey anti-mouse (1:200; Jackson Immunochemicals). All specimens were mounted in anti-fading agent (Vectashield; Vector Laboratories) containing $1 \mu \mathrm{g} / \mu \mathrm{L}$ of DAPI.

For simultaneous assessment of pairing and SYP-1 staining, we first performed a FISH experiment using a probe specific for LG-XL, followed by three 10-min washes in TBST $100 \mathrm{mM}$ Tris- $\mathrm{Cl}$ at $\mathrm{pH} 7.5,136 \mathrm{mM} \mathrm{NaCl}, 25 \mathrm{mM} \mathrm{KCl}$, and $0.1 \%$ Tween 20), a 30-min incubation in TBSB (TBST, 1\% BSA), and an overnight incubation with anti-SYP-1 $(1: 200)$ in TBSB at $4^{\circ} \mathrm{C}$. Slides were then washed three times in TBSB for $15 \mathrm{~min}$, incubated with secondary antibodies for $2 \mathrm{~h}$ at room temperature, and washed three times in TBST for $15 \mathrm{~min}$, followed by mounting as described above.

\section{Image acquisition}

All images were acquired and deconvolved using a DeltaVision Image Restoration System (Applied Precision). Data were collected as series of 15-25 optical sections in increments of 0.2 $\mu \mathrm{m}$ under standard parameters using the SoftWoRx 3.0 program (Applied Precision). Images of pachytene nuclei in wholemounted gonads are the projection of 20 deconvolved images. Images of leptotene-zygotene nuclei, diakinesis nuclei, and spread pachytene nuclei were obtained by projection of 15,10 , and 4 images, respectively.

\section{Time-course analysis of pairing and appearance of RAD-51 foci}

Data for three complete gonads were collected for each genotype and/or each probe used. For each gonad, six to eight stacks of 15-25 optical sections were collected in increments of $0.2 \mu \mathrm{m}$ covering the entire thickness of one layer of nuclei, and an image of the entire gonad was assembled using Photoshop 6.0. The region extending between the first mitotic nuclei and the last pachytene nuclei was divided into five equal-sized zones (45-50 $\mu \mathrm{m}$ in length). FISH signals or RAD-51 foci were then scored by examination of each single nucleus through its volume. FISH signals were considered paired if the distance between the signals was $\leq 0.7 \mu \mathrm{m}$ (MacQueen and Villeneuve 2001). Data for each zone of the three gonads were pooled together, giving a total number of nuclei with paired/unpaired signals, or a total number of nuclei having zero, one, two, three, or more than three RAD-51 foci. The significance of the pairing levels was tested by Fisher's Exact Test (two-tailed $p$ value and $95 \%$ confidence intervals), using InStat 3 software (Graphpad).

\section{$\gamma$-Irradiation experiments}

Twenty-two to twenty-four hours post-L4 hermaphrodites were treated with 5000 rads of $\gamma$-radiation and were fixed and processed for microscopy $4 \mathrm{~h}$ after irradiation following the procedures described above.

\section{Acknowledgments}

We acknowledge the Caenorhabditis Genetic Center, the C. elegans Gene Knockout Consortium, and the Sanger Center for strains and clones; and Adrianna LaVolpe, Anne Villeneuve, and 
Josef Loidl for antibodies. We also thank William Goodyer and Enriquez Martinez-Perez and Anne Villeneuve for communicating unpublished results and for helpful discussions. This work was supported by Canadian Institutes of Health Research (CIHR) and Natural Sciences and Engineering Council (NSERC) research grants.

\section{References}

Aravind, L. and Koonin, E.V. 1998. The HORMA domain: A common structural denominator in mitotic checkpoints, chromosome synapsis and DNA repair. Trends Biochem. Sci. 23: 284-286.

Bhuiyan, H., Dahlfors, G., and Schmekel, K. 2003. Lateral elements inside synaptonemal complex-like polycomplexes in ndt80 mutants of yeast bind DNA. Genetics 163: 539-544.

Brenner, S. 1974. The genetics of Caenorhabditis elegans. Genetics 77: 71-94

Colaiácovo, M.P., MacQueen, A.J., Martinez-Perez, E., McDonald, K., Adamo, A., La Volpe, A., and Villeneuve, A.M. 2003. Synaptonemal complex assembly in C. elegans is dispensable for loading strand-exchange proteins but critical for proper completion of recombination. Dev. Cell 4: 463-474.

Couteau, F., Nabeshima, K., Villeneuve, A.M., and Zetka, M. 2004. A component of C. elegans meiotic chromosome axes at the interface of homolog alignment, synapsis, nuclear reorganization, and recombination. Curr. Biol. 14: 585-592.

Crow, E.L. and Gardner, R.S. 1959. Confidence intervals for the expectation of a Poisson variable. Biometrika 46: 441-453.

Dernburg, A.F., McDonald, J., Moulder, G., Barstead, R., Dresser, M., and Villeneuve, A.M. 1998. Meiotic recombination in C. elegans initiates by a conserved mechanism and is dispensable for homologous chromosome synapsis. Cell 94: 387-398.

Dong, H. and Roeder, G.S. 2000. Organization of the yeast Zip1 protein within the central region of the synaptonemal complex. J. Cell Biol. 148: 417-426.

Fire, A., Xu, S., Montgomery, M.K., Kostas, S.A., Driver, S.E., and Mello, C.C. 1998. Potent and specific genetic interference by double-stranded RNA in Caenorhabditis elegans. Nature 391: 806-811.

Francis, R., Barton, M.K., Kimble, J., and Schedl, T. 1995. gld-1, a tumor suppressor gene required for oocyte development in Caenorhabditis elegans. Genetics 139: 579-606.

Fung, J.C., Rockmill, B., Odell, M., and Roeder, G.S. 2004. Imposition of crossover interference through the nonrandom distribution of synapsis initiation complexes. Cell 116: 795802.

Goldstein, P. 1986. The synaptonemal complexes of Caenorhabditis elegans: The dominant him mutant mnT6 and pachytene karyotype analysis of the $\mathrm{X}$-autosome translocation. Chromosoma 93: 256-260.

— 1987. Multiple synaptonemal complexes (polycomplexes): Origin, structure and function. Cell Biol. Int. Rep. 11: 759-796.

Hodgkin, J., Horvitz, H.R., and Brenner, S. 1979. Nondisjunction mutants of the nematode Caenorhabditis elegans. Genetics 91: 67-94.

Hunter, N. 2003. Synaptonemal complexities and commonalities. Mol. Cell 12: 533-539.

Jang, J.K., Sherizen, D.E., Bhagat, R., Mannheim, E.A., and McKim, K.S. 2003. Relationship of DNA double-strand breaks to synapsis in Drosophila. J. Cell Sci. 116: 3069-3077.

Jantsch, V., Pasierbek, P., Mueller, M.M., Schweizer, D., Jantsch, M., and Loidl, J. 2004. Targeted gene knockout re- veals a role in meiotic recombination for ZHP-3, a Zip3related protein in Caenorhabditis elegans. Mol. Cell. Biol. 24: 7998-8006.

Keeney, S. 2001. Mechanism and control of meiotic recombination initiation. Curr. Top. Dev. Biol. 52: 1-53.

Kelly, K.O., Dernburg, A.F., Stanfield, G.M., and Villeneuve, A.M. 2000. Caenorhabditis elegans msh-5 is required for both normal and radiation-induced meiotic crossing over but not for completion of meiosis. Genetics 156: 617-630.

Leu, J.Y., Chua, P.R., and Roeder, G.S. 1998. The meiosis-specific Hop2 protein of $S$. cerevisiae ensures synapsis between homologous chromosomes. Cell 94: 375-386.

MacQueen, A.J. and Villeneuve, A.M. 2001. Nuclear reorganization and homologous chromosome pairing during meiotic prophase require C. elegans chk-2. Genes \& Dev. 15: 1674 1687.

MacQueen, A.J., Colaiácovo, M.P., McDonald, K., and Villeneuve, A.M. 2002. Synapsis-dependent and -independent mechanisms stabilize homolog pairing during meiotic prophase in C. elegans. Genes \& Dev. 16: 2428-2442.

Martinez-Perez, E. and Villeneuve, A.M. 2005. HTP-1-dependent constraints coordinate homolog pairing and synapsis and promote chiasma formation during C. elegans meiosis. Genes \& Dev. (this issue).

McKim, K.S., Green-Marroquin, B.L., Sekelsky, J.J., Chin, G., Steinberg, C., Khodosh, R., and Hawley, R.S. 1998. Meiotic synapsis in the absence of recombination. Science 279: 876878.

Nabeshima, K., Villeneuve, A.M., and Hillers, K.J. 2004. Chromosome-wide regulation of meiotic crossover formation in Caenorhabditis elegans requires properly assembled chromosome axes. Genetics 168: 1275-1292.

Nasmyth, K. 2005. How do so few control so many? Cell 120: 739-746.

Ollinger, R., Alsheimer, M., and Benavente, R. 2005. Mammalian protein SCP1 forms synaptonemal complex-like structures in the absence of meiotic chromosomes. Mol. Biol. Cell 16: $212-217$.

Page, S.L. and Hawley, R.S. 2004. The genetics and molecular biology of the synaptonemal complex. Annu. Rev. Cell Dev. Biol. 20: 525-528.

Pasierbek, P., Jantsch, M., Melcher, M., Schleiffer, A., Schweizer, D., and Loidl, J. 2001. A Caenorhabditis elegans cohesion protein with functions in meiotic chromosome pairing and disjunction. Genes \& Dev. 15: 1349-1360.

Pawlowski, W.P., Golubovskaya, I.N., Timofejeva, L., Meeley, R.B., Sheridan, W.F., and Cande, W.Z. 2004. Coordination of meiotic recombination, pairing, and synapsis by PHS1. Science 303: 89-92.

Peoples-Holst, T.L. and Burgess, S.M. 2005. Multiple branches of the meiotic recombination pathway contribute independently to homolog pairing and stable juxtaposition during meiosis in budding yeast. Genes \& Dev. 19: 863-874.

Schwacha, A. and Kleckner, N. 1997. Interhomolog bias during meiotic recombination: Meiotic functions promote a highly differentiated interhomolog-only pathway. Cell 90: 11231135

Sherizen, D., Jang, J.K., Bhagat, R., Kato, N., and McKim, K.S. 2005. Meiotic recombination in Drosophila females depends on chromosome continuity between genetically defined boundaries. Genetics 169: 767-781.

Sym, M. and Roeder, G.S. 1995. Zip1-induced changes in synaptonemal complex structure and polycomplex assembly. I. Cell Biol. 128: 455-466.

Tung, K.S. and Roeder, G.S. 1998. Meiotic chromosome morphology and behavior in zip1 mutants of Saccharomyces cer- 
Couteau and Zetka

evisiae. Genetics 149: 817-832.

Villeneuve, A.M. 1994. A cis-acting locus that promotes crossing over between $X$ chromosomes in Caenorhabditis elegans. Genetics 136: 887-902.

Villeneuve, A.M. and Hillers, K.J. 2001. Whence meiosis? Cell 106: 647-650.

von Wettstein, D., Rasmussen, S.W., and Holm, P.B. 1984. The synaptonemal complex in genetic segregation. Annu. Rev. Genet. 18: 331-413.

Walker, M.Y. and Hawley, R.S. 2000. Hanging on to your homolog: The roles of pairing, synapsis and recombination in the maintenance of homolog adhesion. Chromosoma 109: 3-9.

Zalevsky, J., MacQueen, A.J., Duffy, J.B., Kemphues, K.J., and Villeneuve, A.M. 1999. Crossing over during Caenorhabditis elegans meiosis requires a conserved MutS-based pathway that is partially dispensable in budding yeast. Genetics 153: $1271-1283$.

Zetka, M.C. and Rose, A.M. 1995. The genetics of meiosis in Caenorhabditis elegans. Trends Genet. 11:27-31.

Zetka, M.C., Kawasaki, I., Strome, S., and Muller, F. 1999. Synapsis and chiasma formation in Caenorhabditis elegans require HIM-3, a meiotic chromosome core component that functions in chromosome segregation. Genes \& Dev. 13: $2258-2270$.

Zickler, D. and Kleckner, N. 1998. The leptotene-zygotene transition of meiosis. Annu. Rev. Genet. 32: 619-697. 


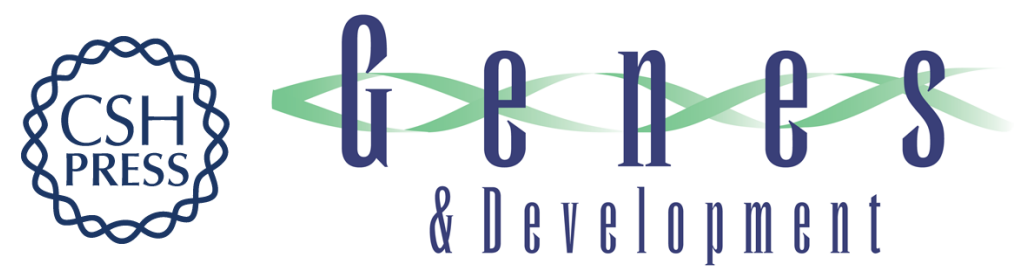

\section{HTP-1 coordinates synaptonemal complex assembly with homolog alignment during meiosis in C. elegans}

Florence Couteau and Monique Zetka

Genes Dev. 2005, 19:

Access the most recent version at doi:10.1101/gad.1348205

Supplemental
Material http://genesdev.cshlp.org/content/suppl/2005/10/27/19.22.2744.DC1

References This article cites 41 articles, 22 of which can be accessed free at:

http://genesdev.cshlp.org/content/19/22/2744.full.html\#ref-list-1

License

Email Alerting

Receive free email alerts when new articles cite this article - sign up in the box at the top

Service

right corner of the article or click here.

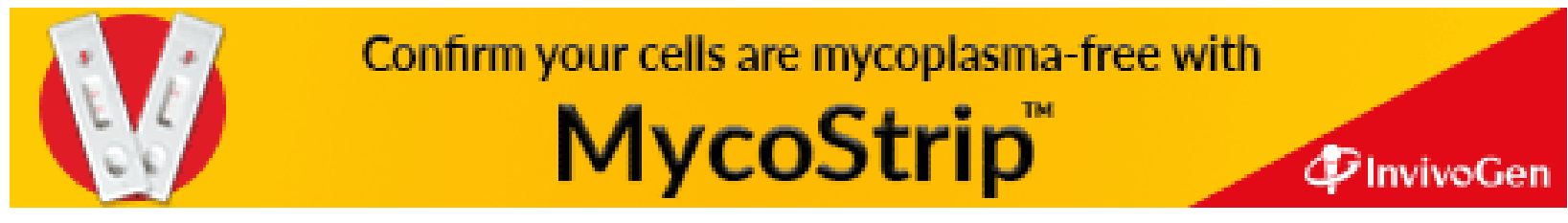

